\title{
Observations on (1-3)- $\beta$-D-glucan detection as a diagnostic tool in endemic mycosis caused by Histoplasma or Blastomyces
}

Fungitell (Associates of Cape Cod) is a new Food and Drug Administration-approved, commercially available assay for the detection of (1-3)- $\beta$-D-glucan (BG), a cell wall polysaccharide of a broad range of fungi, in clinical samples. The presence of BG in serum correlates clinically with certain invasive fungal infections (Odabasi et al., 2004; Ostrosky-Zeichner et al., 2005). However, there are very limited data on the use of Fungitell for serum BG detection to diagnose endemic mycoses. Amongst the dimorphic fungi associated with the endemic mycoses, Histoplasma capsulatum var. capsulatum and Blastomyces dermatitidis share a similar North American distribution. In the vast majority of individuals, infection will cause a transient influenza-like illness or can even be asymptomatic. In certain circumstances, these fungi can cause overt disseminated diseases. Detection of the infection can lag behind a clinically deteriorating patient, owing to inherent diagnostic difficulties (Faggi et al., 2001).

Using the Fungitell assay (http:// www.acciusa.com/pdfs/ fungitell_insert.pdf), we recently tested different serum samples retrieved from patients with active proven histoplasmosis and blastomycosis. Eight out of nine sera collected from five patients with culture-confirmed active disseminated histoplasmosis tested positive for BG (mean value $=859 \mathrm{pg} \mathrm{ml}^{-1}$, range 160-1895, cut-off value 80 ). BG values correlated with Histoplasma antigenuria levels (Pearson $r^{2}=0.88, P=0.03$ ) (Fig. 1). One additional serum tested negative for BG, coming from a human immunodeficiency virus-infected patient with unexplained respiratory symptoms. The diagnosis was made retrospectively when a blood culture collected three months afterwards grew Histoplasma capsulatum.

On the other hand, all four sera collected from four patients with microbiologically proven $B$. dermatitidis infection tested negative for BG. These sera were then subsequently blindly tested by Associates of Cape Cod's Clinical Laboratory Improvement Amendments certified reference laboratory (Beacon Diagnostic). Their results showed a positive BG signal at $97 \mathrm{pg} \mathrm{ml}^{-1}$ in 1 serum, from a chronic disseminated blastomycosis case with multiple skin lesions. The three other sera were negative (Dr Malcome Finkelman, personal communication).

Yeast mycelial dimorphism is a phenomenon observed in many fungi. The endemic mycoses all share this phenotypic switching modulated by environmental conditions, mainly temperature. In $B$. dermatitidis, it has been experimentally demonstrated that thermally induced transition from the mould to the yeast infecting form causes profound depletion in the cell wall BG concentration ratio, passing from 40 to $5 \%$ (Kanetsuna \& Carbonell, 1971). For H. capsulatum though, there is a relative stability in the cell wall BG concentration ratio, passing from 18 to $31 \%$, the mycelial and yeast form, respectively (Kanetsuna et al., 1974). Also, the Histoplasma yeast cell wall has relatively more BG than Blastomyces (Kanetsuna et al., 1974). We think that innate low cell wall BG levels of the Blastomyces infecting yeast may offer a possible explanation for the assay's poor performance in our four patients. Another reason could be the uncommon occurrence of Blastomyces cells in the circulating blood as opposed to Histoplasma.

Our limited data suggest that Fungitell can reliably detect BG in cases of disseminated histoplasmosis. However, the fact that we were not able to detect BG in active Blastomyces infections suggests that BG detection should be used with caution for the general diagnosis of endemic mycosis. Using Fungitell for BG detection has already been proposed as an initial fungal screening test and negative predicted values are reportedly high in the literature, thus preventing uninfected patients being exposed to potential antifungal drug side effects (Digby et al., 2003; Pickering et al.,

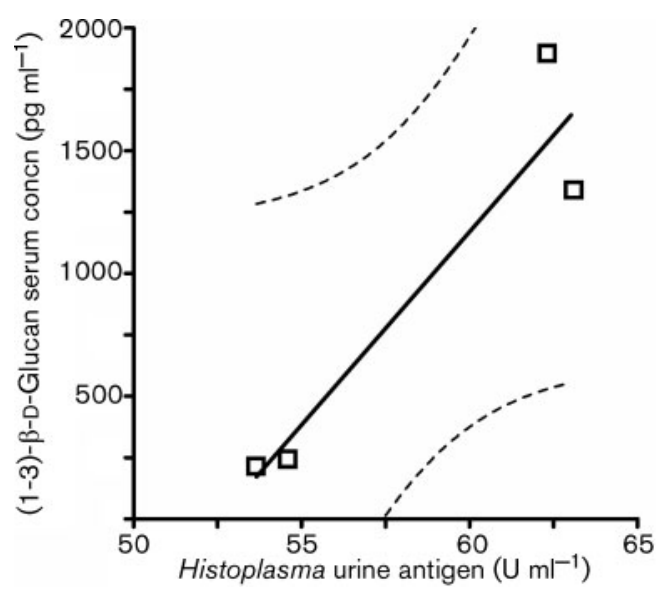

Fig. 1. Individual value ( $\square$ ) of the serum glucan concentration (pg ml${ }^{-1}$ ) compared to the urine Histoplasma antigen (enzyme-linked immunoassay $\mathrm{U} \mathrm{ml}^{-1}$ ) taken simultaneously from the same patient $\left(r^{2}=0.88, P=0.03\right)$. The Pearson linear regression is illustrated by the solid line (dotted lines $95 \%$ confidence intervals). 
2005). It is acknowledged that the test is not of use for detecting rare moulds such as the Zygomycetes (absence of BG synthesis) or Cryptococcus neoformans (encapsulation of the yeast). From the present observations, we suggest that Blastomyces should be added to that list. Although the low number of patients may limit this conclusion, we believe that the clinical implications could be sufficiently serious to alert the medical community. This adds further data on a new and emerging test intended to be used for the non-specific diagnosis of disseminated and life-threatening fungal infections.

\section{G. Girouard, C. Lachance and R. Pelletier}

L'Hotel Dieu de Quebec du CHUQ, 11 Cote du Palais, Quebec, OC G1R 2J6, Canada
Correspondence: R. Pelletier

(rene.pelletier@chuq.qc.ca)

Digby, J., Kalbfleisch, J., Glenn, A., Larsen, A., Browder, W. \& Williams, D. (2003). Serum glucan levels are not specific for presence of fungal infections in intensive care unit patients. Clin Diagn Lab Immunol 10, 882-885.

Faggi, E., Tortoli, E., Bartoloni, A., Corti, G., Pini, G., Simonetti, M. T. \& Campisi, E. (2001). Late diagnosis of histoplasmosis in a Brazilian patient with acquired immunodeficiency syndrome.

Clin Microbiol Infect 7, 48-49.

Kanetsuna, F. \& Carbonell, L. M. (1971). Cell wall composition of the yeast like and mycelial forms of Blastomyces dermatitidis. J Bacteriol 106, 946-948.

Kanetsuna, F., Carbonell, L. M., Gil, F. \& Azuma, I. (1974). Chemical and ultrastructural studies on the cell walls of the yeastlike and mycelial forms of Histoplasma capsulatum. Mycopathol Mycol Appl 54, 1-13.
Odabasi, Z., Mattiuzzi, G., Estey, E., Kantarjian, H., Saeki, F., Ridge, R. J., Ketchum, P. A., Finkelman, M. A., Rex, J. H. \& Ostrosky-Zeichner, L. (2004). $\beta$-D-glucan as a diagnostic adjunct for invasive fungal infections: validation, cutoff development, and performance in patients with acute myelogenous leukemia and myelodysplastic syndrome. Clin Infect Dis 39, 199-205.

Ostrosky-Zeichner, L., Alexander, B. D., Kett, D. H., Vazquez, J., Pappas, P. G., Saeki, F., Ketchum, P. A., Wingard, J., Schiff, R. \& other authors (2005). Multicenter clinical evaluation of the $(1 \rightarrow 3)-\beta$ D-glucan assay as an aid to diagnosis of fungal infections in humans. Clin Infect Dis 41, 654-659.

Pickering, J. W., Sant, H. W., Bowles, C. A., Roberts, W. L. \& Woods, G. L. (2005). Evaluation of a $(1 \rightarrow 3)-\beta$-D-glucan assay for diagnosis of invasive fungal infections. J Clin Microbiol 43, 5957-5962. 